The universe through which Narlikar leads us is more rational than violent. Its phenomena can be discovered, explored and understood by careful analysis. Moreover, the facts and their analysis can be laid open to the scrutiny of the nonspecialist reader.

Precision and economy of expression characterize the narrative as it takes the reader through prerequisite discussions of Newtonian gravitation (and its successful celestial mechanics) to Einstein's theory of gravitation, and then to quite a sophisticated discussion of the formation of black holes and the laws of black-hole physics. The emphasis then waxes astrophysical for a few chapters: stellar evolution, supernovae, neutron stars. By now the reader is ready for pulsars, binary $X$-ray sources, accretion disks, X-ray bursters, and then a chapter on active galaxies and quasars. The material is extremely up-to-date.

Finally, there are two chapters on cosmology. The first is a clear (if conventional) summary of the big bang, synthesis of the chemical elements, its observed echo in the cosmic microwave background. The second, unfortunately, is on the author's own alternative theory, one favoured by virtually no other practising cosmologist. The author is absolutely fair in introducing this chapter as a "nonstandard" and "minority" view; but one is left wondering why he did not apply the same rigorous standard to this chapter as to the previous ones, and delete it in toto (an action which the reader should take on his own).

Several stylistic features set this book apart from the myriad other popular and semi-popular books on "black holes and all that stuff"': its richness of factual detail; the implicit sophistication in its presentation of the scientific process, the interplay of observation with theoretical hypothesis; its uncompromisingly literate and numerate prose. Algebraic formulae are used freely; the reader is assumed to be comtortable with the use of symbols and numbers to represent physical quantities. Further, a sane, methodical organization pervades the whole book. A typical subchapter begins with a question, such as "Does the X-ray source Cygnus X-1 contain a black hole?". Observational facts are then concisely presented, before the author proceeds to discuss the bearing of each of the facts in turn on the question posed.

Probably too sophisticated for a lowest. common-denominator "popular" audience, this book can be recommended strongly to anyone with at least an undergraduate degree in any scientific subject who wants a precise narrative presentation of the excitement of modern astrophysical discovery - and no danger of being talked-down to!

William H. Press is Professor of Astronomy and Physics at Harvard University.

\title{
Self-instruction in high energy physics
}

\section{Maurice Jacob}

Basics of Electron-Positron Collisons. By

Fernand M. Renard. Pp.238. ISBN 2-86332-010-6. (Editions Frontières: 1981.) FF $150, \$ 30$.

WITH the decision to build a Large Electron-Positron storage ring at CERN, the LEP project, a large part of future European activity in high energy physics became invested in the study of electronpositron interactions. Already, a tremendous wealth of results has been collected from the $\mathrm{e}^{+} \mathrm{e}^{-}$colliding ring machines at Stanford (SLAC) and Hamburg (DESY); the LEP project will further extend our ability to investigate the intriguing phenomena thus revealed.

Professor Renard's book on the basics of electron-positron collisions therefore comes at a particularly appropriate time. Many students and experimentalists wish to familiarize themselves with calculations which, over and over again, crop up in the analysis of such collisions. The book should be a good self-teaching guide for that purpose. The author surveys all the standard cases in great detail, giving both explicit formulae and enough of the intermediate steps for their derivation $a b$ initio, while always being meticulous with notation. However, in his gallic approach he has a tendency to start with the most general case, discussing only later simpler particular cases, when the beginner might have found the reverse more helpful. The presentation is nevertheless clear enough to enable the reader to skip the general sections easily and go directly to the specific examples.

Reading this book, and making the effort to derive the many formulae given, should prove a very worthwhile investment of time for the dedicated student with some background in relativistic quantum mechanics. The numerous formulae which it contains will also make it useful to the experimentalist writing a Monte Carlo program to assess the response of a detector to expected reactions. To that extent, the book certainly meets a need.

This said, I should add that the newcomer to the field might feel frustrated at the paucity of figures, when the wealth of experimental results is such that calculated distributions and concepts such as jet structure could have been beautifully illustrated. The surprising absence of graphs or tables is also a serious drawback for the reader wishing to assess which

Yale University Press have recently re-issued Richard Goldschmidt's The Material Basis of Evolution, first published in 1940. The reprint incorporates an introduction by Stephen Jay Gould and, in his words, "commemorates a strong reawakening of interest in Goldschmidt's views among evolutionary biologists". Prices are hbk $\$ 35$, £25; pbk \$12.95, £9. kinematical configurations are the most appropriate for the observation of any particular process. For that reason it is difficult to recommend the book as a reference text; it is pitched, perhaps, too much at the computational level when one could have wished for a more global phenomenological approach. Also, while the scope is quite exhaustive, some sections should be considered merely as brief introductions to particular areas which have come into bloom only recently; this applies especially to the account of quarkonia and jets.

The book thus falls short of providing a balanced, critical survey of research on electron-positron collisions. It should, however, definitely be considered as a useful tool for the dedicated physicist entering this active and exciting field.

Maurice Jacob is a Senior Scientist in the CERN Theory Division, Geneva. He was the organizer of the LEP Summer Study at Les Houches and CERN in 1978 .

\section{Dark reactions}

\section{M.C.W. Evans}

Photosynthesis. Proceedings of the Fifth International Photosynthesis Congress. Edited by G. Akoyunoglou. Six volumes, pp.5,322 excluding indexes. ISBN 0-86689012-2. (Balaban International Science Services: 1982.) \$499. Single volumes also available. Distributors ISS, S. Pomerantz, 2242 Mt Carmel Ave., Glenside, PA 19038; ISS, Couwenhoven 62-49, 3703 HN Zeist, The Netherlands.

I MusT start this review with two admissions: first 1 am an author of a very small part of the books under consideration, and secondly 1 have not read very much of them. However, probably neither is a serious disability. Almost anyone who might review them is an author, and it is highly unlikely that anyone will ever read the entire work. It has 5,322 pages, plus 69 pages of index repeated in each volume, at least 10 million words, and weighs nearly $9 \mathrm{~kg}$. It contains 650 papers written by 850 authors ranging from Abdourakhmanov, l.A., to Zurrer, H., who achieve authorship of between 1 and 13 papers each.

Enough statistics. The books contain the proceedings of a very successful conference held in Halkidiki, in Greece, late in 1980 (see Nature 289, 122; 1981). They appear 18 months after the meeting and the question must be, was their publication necessary or worthwhile? The papers are unreviewed and cover a very wide range of subjects from the biophysical studies of light absorption in the picosecond time range, 